\title{
El Modelo Servqual Y Su Incidencia En EI Nivel De Satisfacción De La Carrera De Economía De La Universidad Nacional De Chimborazo
}

\author{
María González Bautista \\ Eduardo Zurita Moreano \\ Mauricio Zurita Vaca \\ Facultad de Ciencias Políticas y Administrativas \\ UNIVERSIDAD NACIONAL DE CHIMBORAZO
}

doi: 10.19044/esj.2017.v13n25p339 URL:http://dx.doi.org/10.19044/esj.2017.v13n25p339

\begin{abstract}
This article is aimed at evaluating the level of satisfaction that both the internal and external clients of the Economics Career are experiencing. In the case of external clients through the application of the SERVQUAL model, considering that it is based on an analysis of the perception versus the expectation, it also evaluates the quality of service along five unique dimensions: reliability, responsiveness, security, empathy and tangible elements. The main result was that the ability to respond to the clients and provide willing and meaningful help to the users and provide fast service is the weakest parameter of the dimensions that the SERVQUAL Model values. In the case of internal clients, such as teachers, the level of satisfaction was assessed according to a specific methodology.
\end{abstract}

Keywords: Teachers, students, level of satisfaction, SERVQUAL model

\section{Resumen}

El presente trabajo está dirigido a valorar el nivel de satisfacción que tienen los clientes internos y externos de la Carrera de Economía. En el caso de los clientes externos mediante la aplicación del modelo SERVQUAL, considerando que este se basa en un análisis de la percepción versus la expectativa, además este evalúa la calidad de servicio a lo largo de cinco dimensiones: fiabilidad, capacidad de respuesta, seguridad, empatía y elementos tangibles. Como principal resultado se obtuvo que la capacidad de respuesta es decir la disposición y voluntad para ayudar a los usuarios y proporcionar un servicio rápido constituya el parámetro más débil de las dimensiones que valora el modelo SERVQUAL. En el caso de los clientes internos es decir los docentes se determinó el nivel de satisfacción en función 
a una metodología propia.

Palabras clave: docentes, estudiantes, nivel de satisfacción, modelo SERVQUAL

\section{Introducción}

Según el Consejo de Evaluación, Acreditación y Aseguramiento de la Calidad de la Educación Superior (CEAACES, 2016), la evaluación de desempeño institucional de las instituciones de educación superior, se ha orientado a generar los elementos para la calidad, con el objetivo de proceder a la depuración y al mejoramiento del Sistema Nacional de Educación Superior.

Según en el Art. 173. de la LOES "Las universidades, escuelas politécnicas, institutos superiores técnicos, tecnológicos, pedagógicos, de artes y conservatorios superiores del país, tanto públicos como particulares, sus carreras y programas, deberán someterse en forma obligatoria a la evaluación interna y externa, a la acreditación, a la clasificación académica y al aseguramiento de la calidad". (Ley Orgánica de Educación Superior , 2016). De acuerdo al informe entregado por el CEAACES en septiembre 2015, las universidades se categorizaron, de acuerdo al cumplimiento de los indicadores en las siguientes categorías: A, B, C y D.

Esta categorización de las universidades pone en evidencia la necesidad de valorar la calidad de los servicios educativos para garantizar su existencia y por ende carreras y programas en el mercado educativo. Según la norma ISO 9000; 2005, la calidad se refiere al grado de cumplimiento de necesidades sobrentendidas y a veces ineludibles por parte de un ente que ofrece un legajo de atributos que le diferencian. (International Organization for Standardization, 2005)

"El principio de calidad consiste en la búsqueda constante y sistemática de la excelencia, la pertinencia, producción óptima, transmisión del conocimiento y desarrollo del pensamiento mediante la autocrítica, la crítica externa y el mejoramiento permanente." (Ley Orgánica de Educación Superior, 2010)

La calidad en un principio es subjetiva, al estar definida por cada usuario o cliente, pero la misma a través de la identificación de factores específicos, dimensiones y criterios se la puede medir y cuantificar para poder crear un estándar el cual llene la mayor cantidad de expectativas. (Camisón, Cruz, \& González, 2006)

Según (Rebolloso, Salvador, Fernández, \& Cantón, 2005) La coyuntura social y económica han obligado a las instituciones a establecer "compromisos éticos" que les permitan igualarse en prestigio con otro tipo 
de organizaciones. En el caso de Ecuador cumplir con estándares que le permitan ubicarse en la categoría "A".

Con el proposito de valorar la calidad del servicio se ha tomado en consideración el modelo SERVQUAL el mismo que según (Pineda, Estrada, \& Parra, 2011) valora la brecha entre expectativas y percepción de la calidad del servicio a lo largo de cinco dimensiones. En tal virtud, (Salvador, 2004) explica que las dimensiones son: elementos tangibles, fiabilidad, capacidad de respuesta, seguridad, empatía.

Según (Quevedo \& Andalaft, 2008) las dimensiones de la escala SERVQUAL son:

a) Fiabilidad: se refiere a la habilidad para realizar el servicio de modo cuidadoso y fiable.

b) Capacidad de Respuesta: se refiere a la disposición y voluntad para ayudar a los usuarios y proporcionar un servicio rápido.

c) Seguridad: hace referencia a los conocimientos y atención mostrados por los empleados y sus habilidades para concitar credibilidad y confianza.

d) Empatía: hace referencia a la atención personalizada que dispensa la organización a sus clientes.

e) Elementos Tangibles: Son las instalaciones físicas, equipos, personal y materiales de comunicación.

Por su parte, (Coulthard, 2004) manifiesta que el modelo SERVQUAL está diseñado con el fin de evaluar la calidad del servicio brindado al usuario, desde su perspectiva, teniendo como propósito principal para su base que un servicio de calidad viene dado cuando las percepciones equiparan o sobresalen sobre las expectativas que el cliente se hizo en relación a un producto o servicio.

En la actualidad las organizaciones (Morales, Hernández, \& Blanco, 2009) se enfocan en la calidad de los servicios y esto se debe principalmente al concepto de excelencia que conlleva: aumentar la incorporación de nuevos clientes, fidelizar a los clientes potenciales, ofrecer oportunidades objetivas para la mejora y el desarrollo organizacional, optimizar la relación costes/beneficios. En ese sentido (Rebolloso, Fernández , \& Cantón, 2001) destacan la importancia de generar un servicio de calidad que promueva la fidelización de los clientes.

De nada serviría disponer de altos niveles de gestión de calidad si no existieran receptores del producto. Por tanto, conocer cómo valoran los clientes el servicio ofrece información valiosa para construir tecnologías de gestión que permitan un funcionamiento eficaz. (Martínez-Tur, Peiró , \& Ramos , 2001)

Otro punto importante que se debe mencionar en base a (Cronin \& Taylor, 1992) son las discrepancias a nivel conceptual y operacional sobre la 
interpretación entre calidad del servicio y la satisfacción de los usuarios lo cual no está claramente identificado. (Bolton \& Drew, 1991) Es importante indicar las cuestiones críticas relacionadas con la composición de sus elementos, los altos grados de polarización en varios elementos, las imperfecciones en el número de dimensiones y el uso de los mismos elementos para analizar la calidad del servicio y el nivel de satisfacción de los clientes. (Churchill \& Surprenant, 1982)

Cabe destacar que las funciones sustantivas de la universidad ecuatoriana, previstas en la (Constitución de la República del Ecuador, 2008) son: docencia, investigación y vinculación con la colectividad, a las cuales se agrega la gestión administrativa que se constituye en apoyo de las anteriores.

Es fundamental que en todas las áreas anteriormente mencionadas exista calidad y un nivel de satisfacción adecuado por parte de los usuarios ya sean estos internos o externos, lo que implica contar con infraestructura, empatía, capacidad de respuesta, fiabilidad, seguridad, todas estas dimensiones del SERVQUAL.

\section{Métodos y materiales}

El estudio se llevó a cabo en la Carrera de Economía de la Universidad Nacional de Chimborazo, contempló dos etapas: en primera instancia se valoró el nivel de satisfacción que tenían los clientes externos (estudiantes) a través del modelo SERVQUAL y en segunda instancia se valoró el nivel de satisfacción de los clientes internos (docentes), mediante un cuestionario personalizado para la población objetivo.

En lo referente al análisis de los clientes externos se utilizó el cuestionario SERVQUAL. Esta metodología requiere de un instrumento a manera de escala de medición en relación a la calidad percibida en un servicio, formado a partir de las dimensiones de calidad, las cuales son calificadas en relación a la percepción y a la expectativa. (Camisón, Cruz, \& González, 2006)

El cuestionario SERVQUAL utiliza la escala Likert, (Malave, 2007) la describe en base a la siguiente ponderación: Totalmente en desacuerdo con una ponderación de 1; Bastante en desacuerdo con una ponderación de 2; Ni de acuerdo ni en desacuerdo con una ponderación de 3; Bastante en acuerdo con una ponderación de 4; Totalmente en acuerdo con una ponderación de 5. El significado de la escala LIKERT para interpretar la satisfacción del cliente se basa en el siguiente cuadro: 
Cuadro 1: Significado de la escala LIKERT

\begin{tabular}{ccc}
\hline NIVEL DE LIKERT & SIGNIFICADO & $\begin{array}{c}\text { SATISFACCIÓN DEL } \\
\text { CLIENTE }\end{array}$ \\
\hline $\mathbf{1}$ & Extremadamente Insatisfecho & $0-20$ \\
\hline $\mathbf{2}$ & Insatisfecho & $20-40$ \\
\hline $\mathbf{3}$ & Neutro & $40-60$ \\
\hline $\mathbf{4}$ & Satisfecho & $60-80$ \\
\hline $\mathbf{5}$ & Extremadamente Satisfecho & $80-100$ \\
\hline
\end{tabular}

Fuente: (Hernandez, et al 2002)

Para evaluar la calidad percibida se plantean estas dimensiones generales y se definen que dicha percepción es consecuencia de la diferencia para el consumidor entre lo esperado y lo percibido. El modelo SERVQUAL, con el estudio de los cinco gaps, analiza los principales motivos de la diferencia que llevan a un fallo en las políticas de calidad de las organizaciones. (Duque, 2005)

Este modelo es uno de los más usados, aunque posee muchas críticas en relación a su utilización, en cuanto a que las dimensiones en ocasiones no cubren todos los parámetros para medir la calidad del servicio de una organización. En ese sentido para valorar de mejor forma el nivel de satisfacción del cliente y bajo el criterio de los autores se contempló una dimensión adicional denominada "Satisfacción específica" la misma que toma en consideración aspectos como la metodología aplicada en los procesos de enseñanza aprendizaje, entre otros.

Según (Parasuraman, A., Valerie A. , Zeithaml , \& Leonard L. , 1993) Para determinar el nivel de la calidad de servicio considerando el modelo SERVQUAL (aplicado a clientes externos) se tomó en consideración la siguiente formula:

Dónde:

$$
Q_{1 i}=\sum_{j=1}^{k}\left(P_{i j}-E_{i j}\right)
$$

$\mathrm{Q}=$ calidad percibida global del elemento $\mathrm{i}$;

$\mathrm{k}=$ número de atributos;

$\mathrm{Pij}=$ percepción del resultado del estímulo i con respecto al atributo j;

Eij = expectativa del atributo $\mathrm{j}$ para el elemento i.

Para el presente estudio se utilizaron 2 poblaciones, una para clientes internos y otra a clientes externos, considerando que se aplicaron instrumentos de recolección de información diferentes, la población de clientes internos en este caso fueron 21 docentes, y los clientes externos fueron los estudiantes de la carrera de Economía que ascendieron a 225 encuestas. 
Además en el caso de los clientes externos se aplicaron las encuestas por dos ocasiones. La una para medir la percepción del servicio, y la otra para medir la expectativa del mismo.

Al medir la satisfacción de los clientes internos no se puede aplicar todas las dimensiones del modelo SERVQUAL, considerando que los clientes internos, están de cierta manera cautivos e incluso obligados, por lo que las dimensiones a medir debieron variar. (Camisón, Cruz, \& González, 2006)

No existe una escala aceptada como universal para evaluar la satisfacción del cliente interno, por lo que se realizó un instrumento en relación a las necesidades organizacionales, dependiendo de los compontes a medir. En ese sentido para analizar el nivel de satisfacción de los docentes de la Carrera se aplicó otra metodología en función a sus necesidades y demandas.

\section{Resultados}

El servicio educativo se valoró desde dos enfoques: En primera instancia desde los clientes externos: "El cliente externo es la persona que no pertenece a la empresa y solicita satisfacer una necesidad (bien o servicio)." (Ameca, 2014)

En segunda instancia desde el enfoque de los clientes internos: "Es el que se encuentra produciendo un bien o servicio dentro de la organización, trabajando en unidades o departamentos y provee servicios a los otros departamentos, es decir son un cliente o proveedor al mismo tiempo". (Pérez, 2006).

\section{CLIENTES EXTERNOS}

En lo referente al cliente externo, el nivel de satisfacción de los estudiantes de la carrera de economía de la UNACH, en base a la aplicación del modelo SERVQUAL los resultados son los siguientes:

Cuadro 2: Nivel de Satisfacción de los estudiantes de la Carrera de Economía a partir de la aplicación del Modelo SERVQUAL.

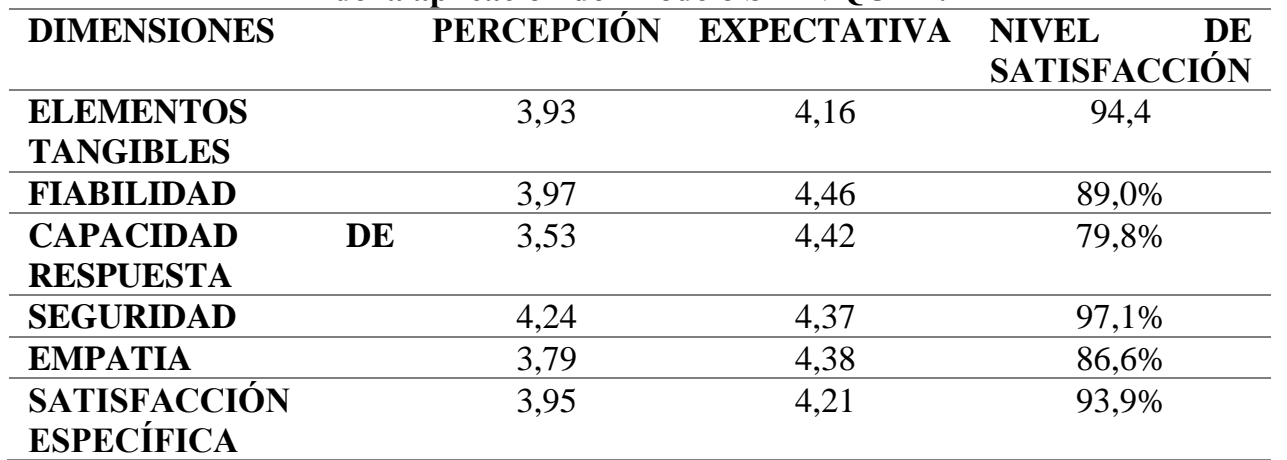

Fuente: Encuestas aplicadas a los estudiantes de la Carrera de Economía de la UNACH.

Elaboración: Autores 
Gráfico 1: Nivel de Satisfacción de los estudiantes de la Carrera de EconomíaAplicación del Modelo SERVQUAL.

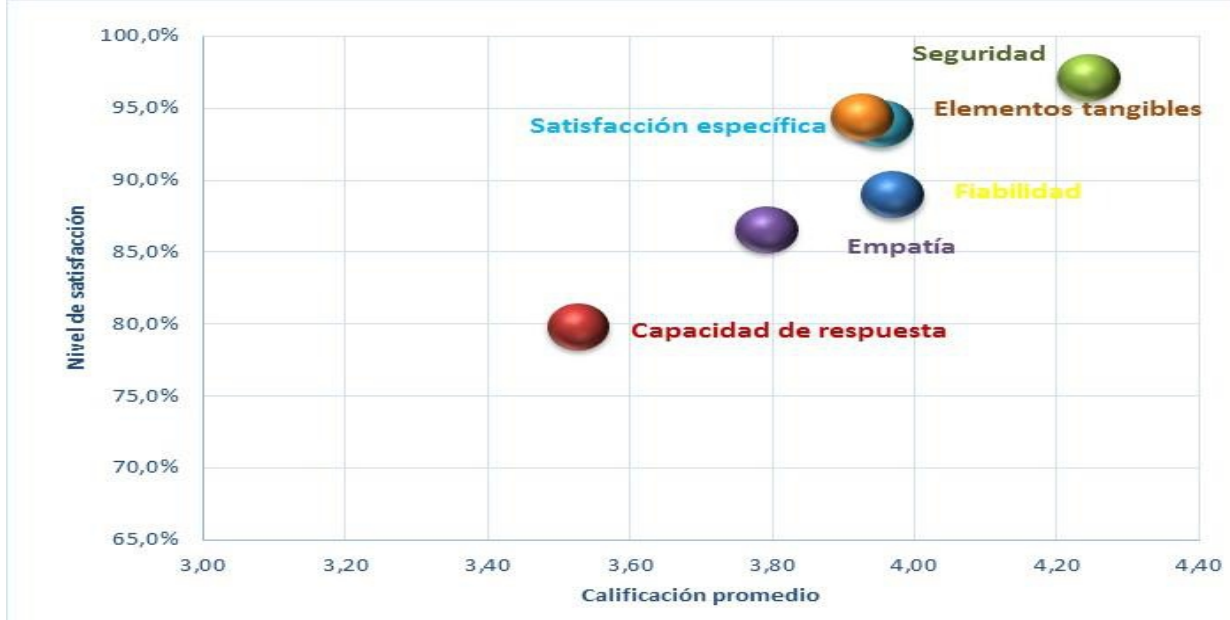

Fuente: Encuestas aplicadas a los estudiantes de la Carrera de Economía de la UNACH.

Elaboración: Autores

Se puede apreciar que existe una percepción con respecto a la dimensiones del SERVQUAL en elementos tangibles de 3,93; fiabilidad 3,97; Capacidad de respuesta 3,53; Seguridad 4,24; Empatía 3,79 y finalmente en satisfacción específica 3,79 , comparado con la expectativa es decir cuánto esperaba el estudiante recibir del servicio se da lugar al nivel de satisfacción, se puede notar que en las dimensiones de elementos tangibles tienen el 94,4\%; fiabilidad tienen el 93,2\%; capacidad de respuesta el 79,8\%; seguridad el 97,1\%; Empatía el 86\% y finalmente en satisfacción específica el 93,9\%.

\section{ELEMENTOS TANGIBLES}

En un contexto general se puede apreciar que los elementos tangibles es decir instalaciones físicas, condiciones del equipo y el resto de instalaciones en que se presta el servicio educativo, se ha obtenido una percepción promedio de 4,28 con respecto a la expectativa que es de 4,52 lo que da un nivel de satisfacción del $95 \%$.

Los principales resultados que se derivan de esta dimensión son: Con respecto a las instalaciones físicas que utiliza la Carrera de Economía y si estas son adecuadas para los procesos de enseñanza aprendizaje se ha obtenido un nivel de satisfacción del 98\%.

En lo concerniente a las aulas se ha alcanzado un nivel de satisfacción del 97\%, cabe destacar que las aulas se utilizan en dos jornadas, primero a 
quinto semestre los estudiantes reciben clases en la mañana, de sexto semestre en adelante los estudiantes reciben clases en la tarde.

Las baterías sanitarias que dispone la Carrera de Economía son adecuadas, la percepción es de 3,34 con respecto a la expectativa de 3,76 por lo que se ha obtenido un nivel de satisfacción del $89 \%$, que representa el nivel de satisfacción más baja dentro de esta dimensión. Sin embargo de manera general (toda la dimensión) se puede deducir que la Carrera de Economía de la UNACH cuenta con una infraestructura moderna que satisface las demandas de los clientes externos.

\section{Gráfico 2: Elementos Tangibles}

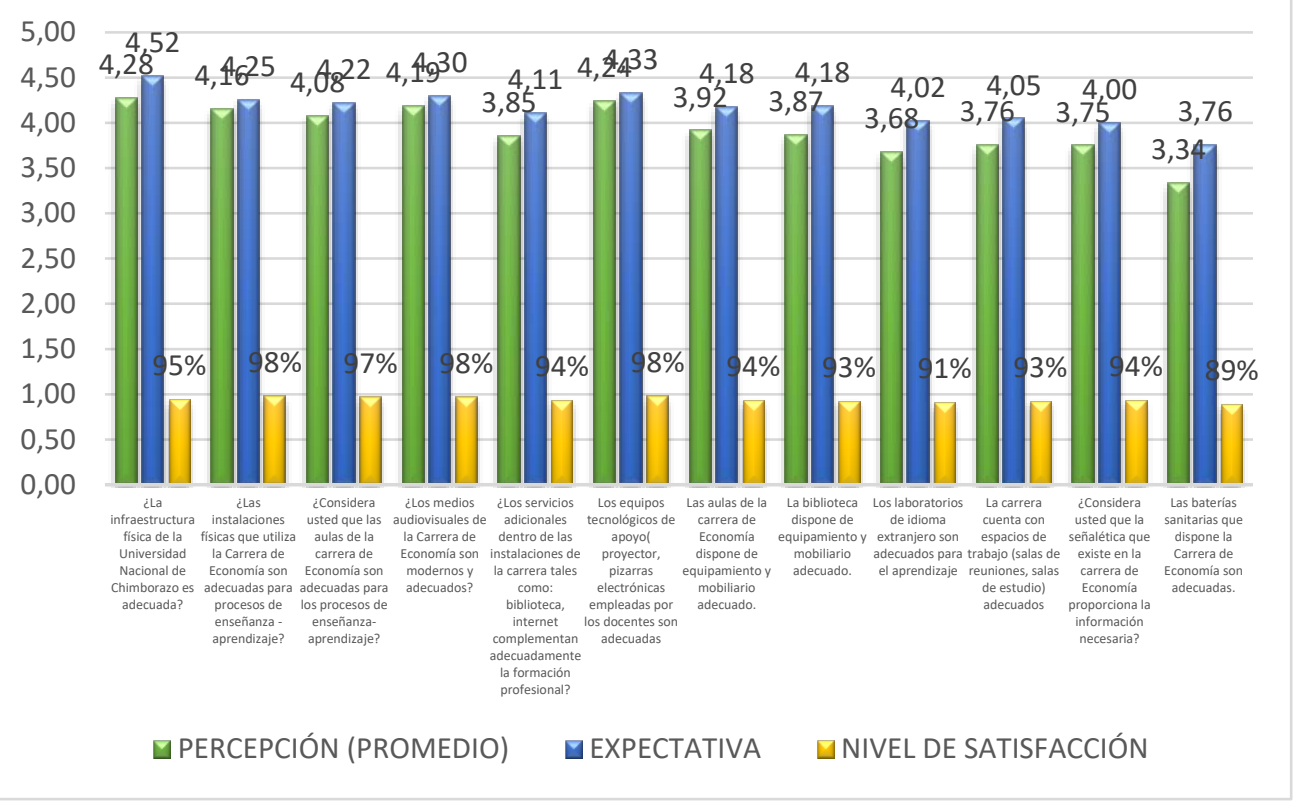

Fuente: Encuestas aplicadas a los estudiantes de la Carrera de Economía de la UNACH.

Elaboración: Autores

\section{FIABILIDAD}

La fiabilidad se refiere a la efectividad es decir obtener el servicio mediante un proceso correcto que cumpla las expectativas para el que fue diseñado. En un contexto general (toda la dimensión) el nivel de satisfacción es de $93,2 \%$;

$\mathrm{Al}$ desagregar esta dimensión entre los principales resultados que se obtuvieron están: La carrera cuenta con personal administrativo adecuado calificado acorde a sus competencias y funciones, el nivel de satisfacción fue del $96 \%$, con respecto a que los docentes demuestran interés y disponibilidad para aclarar dudas fuera de clases, el nivel fue de $95 \%$.

En lo referente a que los funcionarios de la carrera demuestran interés para solucionar problemas con respecto a trámites de los estudiantes. Este aspecto refleja el nivel más bajo con el $87 \%$, respecto a que si la carrera 
cuenta con una malla curricular acorde a las exigencias del mercado laboral se ha obtenido un nivel de satisfacción del $93 \%$.

\section{Gráfico 3: Fiabilidad}

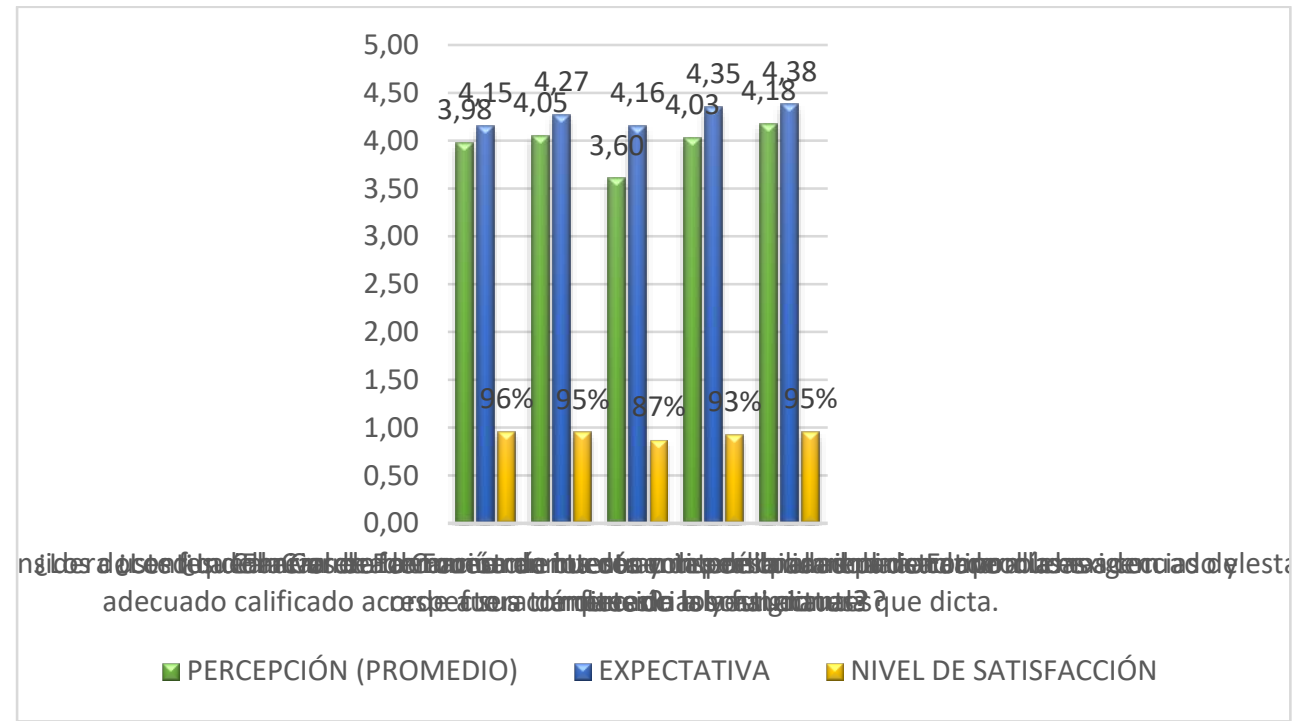

Fuente: Encuestas aplicadas a los estudiantes de la Carrera de Economía de la UNACH.

Elaboración: Autores

\section{CAPACIDAD DE RESPUESTA}

La capacidad de respuesta evalúa si la cantidad de empleados es suficiente para la atención así como los tiempos de espera poder recibir el servicio solicitado, con respecto a esta dimensión el nivel de satisfacción general fue de 79,8\%. Siendo el nivel de satisfacción más bajo con respecto a las otras dimensiones.

Dentro de esta dimensión los aspectos más destacados son: Los funcionarios de la carrera son ágiles y eficientes en los diferentes trámites requeridos por los estudiantes, se ha alcanzado un nivel de satisfacción del $79 \%$.

Otro aspecto fue la capacidad de respuesta por parte de las secretarias es ágil y eficiente obteniendo un nivel de satisfacción del $64 \%$. Un punto importante a resaltar es que si los trámites y gestiones dentro de la Universidad y de la Carrera de Economía son atendidos y resueltos en tiempos adecuados se ha obtenido un nivel de satisfacción del $78 \%$. 
Gráfico 4: Capacidad de Respuesta

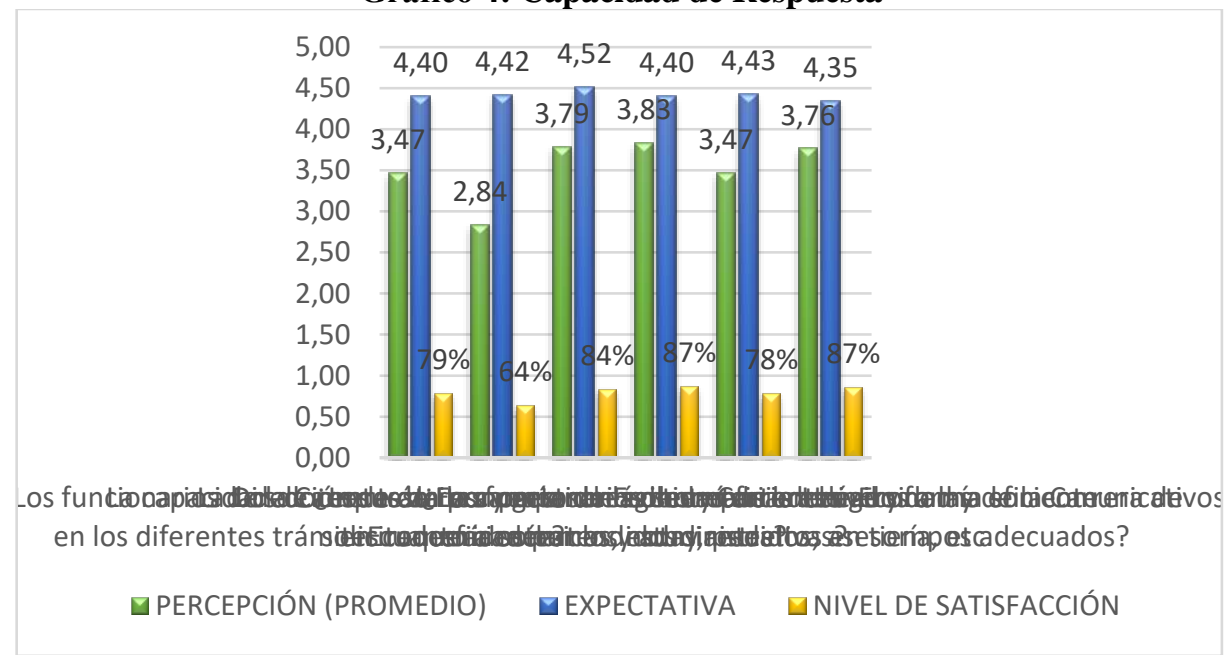

Fuente: Encuestas aplicadas a los estudiantes de la Carrera de Economía de la UNACH. Elaboración: Autores

\section{SEGURIDAD}

La Seguridad se refiere a la competencia del personal, la confianza que generan al momento de prestar el servicio, el nivel de satisfacción general en esta dimensión fue de $97,1 \%$.

En esta dimensión un aspecto a destacar es el nivel de satisfacción de la preparación académica de los docentes con un 98\%. Otro aspecto analizado fue que los profesores asisten a clases regularmente con un nivel de satisfacción del 97\%. Finalmente, la Carrera de Economía le garantiza conocimientos y competencias para un adecuado desenvolvimiento laboral, alcanzando un nivel de satisfacción del 96\%.

\section{Gráfico 5: Seguridad}

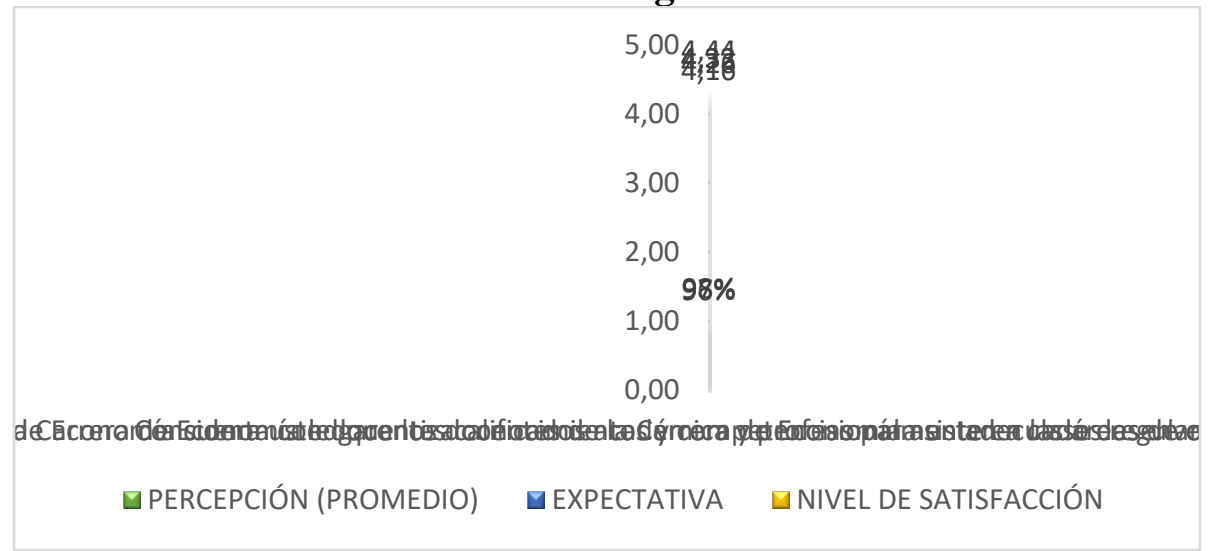

Fuente: Encuestas aplicadas a los estudiantes de la Carrera de Economía de la UNACH.

Elaboración: Autores 


\section{EMPATÍA}

La empatía se refiere a la medición del trato, atención e interés que el personal de la institución manifiesta hacia los usuarios de la misma y el trato individualizado que se da, el nivel de satisfacción que se obtuvo fue de $86 \%$. Cabe destacar que para que se dé la empatía, se requiere de una buena comunicación entre la organización y el usuario.

Al desarticular la dimensión se obtuvieron los siguientes resultados: Los docentes realizan tutorías académicas de forma permanente y planificada tuvo un nivel de satisfacción del $83 \%$.

Con respecto a la atención que se brinda en la biblioteca seobtuvo un nivel de satisfacción del 79\%. Se puede notar que de todos los aspectos anteriormente citados en esta dimensión esta fue la menos valorada por los estudiantes.

\section{Gráfico 6: Empatía}

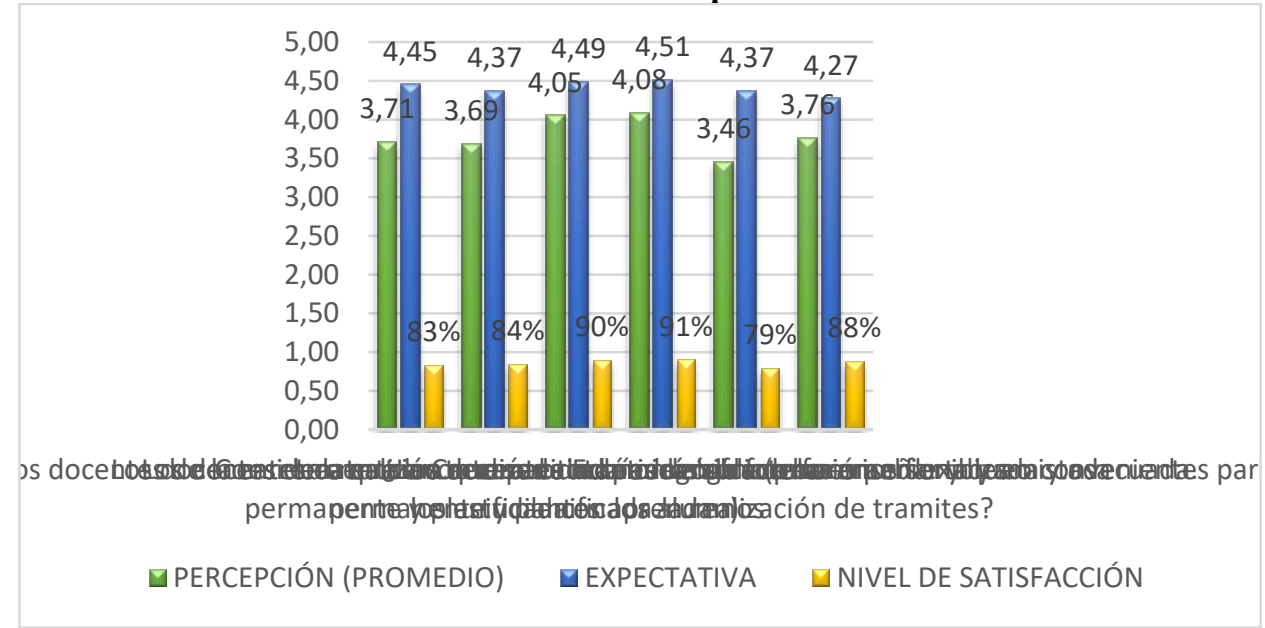

Fuente: Encuestas aplicadas a los estudiantes de la Carrera de Economía de la UNACH.

Elaboración: Autores

\section{SATISFACCIÓN ESPECÍFICA}

En lo referente a satisfacción específica, se obtuvo un nivel de satisfacción de 93,9\%. Aquí se analizaron aspectos tales como, los docentes hacen uso de las TICS en forma permanente y adecuada con un nivel del 96\%. Las metodologías empleadas por los docentes un nivel de satisfacción del $95 \%$, el nivel de investigación aplicado por los docentes en las actividades curriculares el $95 \%$

En lo concerniente a las prácticas, los conocimientos impartidos en las aulas de clase son adecuados evidenciando un nivel de satisfacción del $90 \%$. Finalmente, considera usted que los docentes son objetivos al momento de evaluar los procesos de aprendizaje, en este aspecto se ha obtenido un nivel de satisfacción del $98 \%$. 


\section{Gráfico 7: Satisfacción Específica}

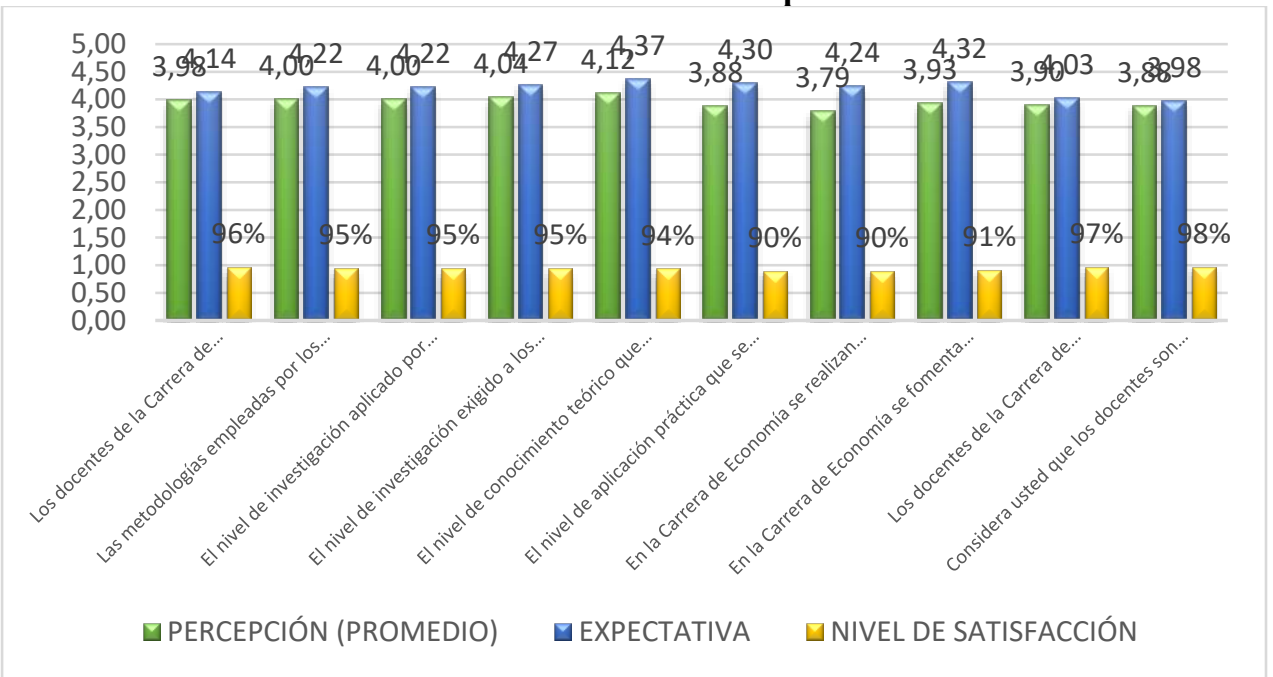

Fuente: Encuestas aplicadas a los estudiantes de la Carrera de Economía de la UNACH.

Elaboración: Autores

\section{CLIENTES INTERNOS}

En lo que se refiere al nivel de satisfacción de los docentes, los principales aspectos fueron los siguientes:

El equipamiento de las aulas es adecuado; bastante en desacuerdo 9,5\%, ni de acuerdo ni en desacuerdo 19\%; bastante de acuerdo 38,1\%; totalmente de acuerdo $33,3 \%$.

La implementación de la biblioteca es: totalmente en desacuerdo 9,5\%; bastante en desacuerdo $28,6 \%$; ni de acuerdo ni en desacuerdo $38,1 \%$; bastante de acuerdo 19\%; totalmente de acuerdo 4,8\%.

La comunicación intrafacultad es adecuada con las calificaciones de: bastante en desacuerdo 4,8\%; ni de acuerdo ni en desacuerdo 28,6\%; bastante de acuerdo 52,4\%; totalmente de acuerdo $14,3 \%$.

La remuneración recibida por sus actividades docentes es adecuada: bastante en desacuerdo 9,5\%; ni de acuerdo ni en desacuerdo 33,3\%; bastante de acuerdo $23,8 \%$; totalmente de acuerdo $33,3 \%$.

Temas de capacitación académica recibidos con las valoraciones de: totalmente en desacuerdo 4,8\%; bastante en desacuerdo 4,8\%; ni de acuerdo ni en desacuerdo 38,1\%; bastante de acuerdo 19\%; totalmente de acuerdo $33,33 \%$.

\section{Conclusion}

Respecto a los estudiantes (clientes externos), se puede apreciar que existe un nivel de satisfacción en la dimensión de elementos tangibles del 
94,4\%; fiabilidad el 93,2\%; capacidad de respuesta el 79,8\%; seguridad el 97,1\%; empatía el 86\% y finalmente en satisfacción específica 93,9\%.

En el caso de los estudiantes, la capacidad de respuesta constituye la dimensión más débil por lo que es preciso mejorar los tiempos de espera al momento de recibir el servicio.

En referencia a los docentes (clientes internos) se encuentran insatisfechos con los espacios trabajo para realizar sus actividades el 9,5\% y los que estan de acuerdo representan el 71,4\%; en lo referente a la biblioteca el $38,1 \%$ esta en desacuerdo y el $23,8 \%$ esta de acuerdo; sobre la comunicación dentro de la facultad el 4,8\% esta en desacuerdo y el $66,7 \%$ esta satisfecho; al referirse sobre la remuneración que perciben el 9,5\% no esta satisfecho y el 57,1\% esta de acuerdo; y finalmente con respecto a la capacitación prestada por la institución se obtuvo el siguiente resultado, $9,6 \%$ estaba en desacuerdo y el 52,3\% manifesto que estan satisfechos, ya que manifiestan la importancia de la capacitación en áreas específicas de estudio.

\section{References:}

1. Ameca, J. (2014). http://www.gestiopolis.com/. Obtenido de Cliente interno $y$ externo en una organizació: http://www.gestiopolis.com/cliente-interno-y-externo-en-unaorganizacion/

2. Bolton, R., \& Drew, J. (1991). A multistage model of customers assessments.

3. Consejo de Evaluación, Acreditación y Aseguramiento de la Calidad de la Educación Superior CEAACES. (2016). http://www.ceaaces.gob.ec/sitio/acreditacion-y-categorizacion/.

Obtenido de http://www.ceaaces.gob.ec/sitio/acreditacion-ycategorizacion/.

4. Churchill, G., \& Surprenant, C. (1982). An investigation into the determideterminants.

5. Coulthard, L. (2004). Measuring service quality: A review and critique of research using SERVQUAL.

6. Cronin, J., \& Taylor, S. (1992). Measuring service quality: A reexamination and extension. Journal of Marketing.

7. Camisón, C., Cruz, S., \& González, T. (2006). Gestión de la Calidad: conceptos, enfoques, modelos y sistemas. Madrid: Pearson Educación S. A.

8. Duque, E. (2005). Revisión del concepto de calidad del servicio y sus modelos de medición.

9. Hernández, R., Fernández, C., \& Baptista, P. . (2002). Metodología de la Investigación. México, D.F: McGraw Hill. 
10. International Organization for Standardization. (2005). Sistemas de gestión de la calidad - Fundamentos y vocabulario. Ginebra: Secretaría Central de ISO.

11. Ley Orgánica de Educación Superior . (2016). http://www.ceaaces.gob.ec/sitio/acreditacion-y-categorizacion/.

Obtenido de http://www.ceaaces.gob.ec/sitio/acreditacion-ycategorizacion/.

12. Martínez-Tur, Peiró , \& Ramos . (2001). Calidad del servicio y satisfacción del cliente.

13. Morales, V., Hernández, A., \& Blanco, Á. (2009). EVALUACIÓN DE LA CALIDAD EN ORGANIZACIONES DEPORTIVAS: ADAPTACIÓN DEL MODELO SERVQUAL. 18(2).

14. Moreno, C. (2012). "Evaluación de la gestión académica y administrativa de la escuela de economía de la universidad nacional de chimborazo y los efectos en la formación de sus egresados. Período 2008-2009. Propuesta alternativa".

15. Parasuraman, A., Valerie A. , Zeithaml , \& Leonard L. . (1993). Calidad Total de Gestión de los Servicios: cómo lograr el equilibrio entre las percepciones y expectativas de los consumidores. Madrid: Díaz de los Santos. Obtenido de http://www.itson.mx: http://www.itson.mx/publicaciones/pacioli/Documents/no59/consulto ria/servqual.pdf

16. Pérez, V. (2006). Calidad total en la atención al cliente. Pautas para garantizar la excelencia en el servicio. España: Ideaspropias Editorial. Vigo.

17. Quevedo, A., \& Andalaft, A. (2008). Evaluación y propuesta de mejoras de un modelo de atención de usuarios en un servicio público. Revista economía y administración $\mathrm{n}^{\circ} 71$.

18. Pineda, U., Estrada, M., \& Parra, C. (2011). Aplicación del modelo Servqual y herramientas de ingeniería de la calidad para la planificación del servicio en la Biblioteca Central de la Universidad de Antioquia.

19. Rebolloso, E., Salvador, C., Fernández, B., \& Cantón, P. (2005). Análisis y ampliación del SERVQUAL en los servicios universitarios.

20. Salvador, C. (2004). La matriz humana de los servicios universitarios. 